\title{
Selectivity and Potency of Natural Product Pim Kinase Inhibitors Identified By in Silico Docking
}

\author{
Michael Russell
}

Brigham Young University

Nicholas Fazio ( $\nabla$ nicholasf.fazio@gmail.com )

Brigham Young University https://orcid.org/0000-0002-8325-6778

Jace Webster

Brigham Young University-Provo: Brigham Young University

Marc Hansen

Brigham Young University-Provo: Brigham Young University https://orcid.org/0000-0001-6588-2030

\section{Research Article}

Keywords: Proviral Integration site, Maloney murine leukemia virus kinase 3, PIM3, computational screen, diosgenin glycoside, biflavonoid

Posted Date: February 15th, 2021

DOI: https://doi.org/10.21203/rs.3.rs-188695/v1

License: (1) (1) This work is licensed under a Creative Commons Attribution 4.0 International License. Read Full License

Version of Record: A version of this preprint was published at Medicinal Chemistry Research on March 17th, 2021. See the published version at https://doi.org/10.1007/s00044-021-02713-w. 


\section{SELECTIVITY AND POTENCY OF NATURAL PRODUCT PIM KINASE INHIBITORS IDENTIFIED BY IN SILICO DOCKING}

MICHAEL H. RUSSELL, NICHOLAS F. FAZIO, JACE WEBSTER, \& MARC D.H. HANSEN*

Words: 3,950

Department of Physiology and Developmental Biology

Brigham Young University

4005 LSB

Provo, UT 84602

USA

* Corresponding author:

Phone: (801) 422-4998

$\checkmark$ marchansen@byu.edu 


\section{ABSTRACT}

PIM3 (Proviral Integration site for Maloney murine leukemia virus kinase 3) is a proto-oncogene with serine/threonine kinase activity that prevents apoptosis, promotes cell survival, and stimulates protein translation. Additionally, PIM3 functions in inflammation and immunity pathways. PIM3 inhibitors are being developed to treat cancer and inflammation-related disorders. Here we screen a 98,000 compound virtual library of natural products to identify those that are predicted to fit in the ATP site of PIM3. Since the structure of PIM3 has not been determined experimentally, we performed molecular structure prediction using the SWISSMODEL tool to generate a PIM3 model structure for in silico screening. Compounds predicted to fit the ATP binding site of PIM3 were validated using biochemical assays, revealing activity against PIM3 for all 8 candidates, with potencies mostly in the micromolar range. We tested several analogs of two validated candidates, the diosgenin glycoside dioscin and the biflavonoid hinokiflavone. Among five dioscin analogs, three exhibit similar potency against PIM3, and with some selectivity for PIM3 versus PIM1 and 2. Meanwhile, 3 of seven biflavonoid analogs exhibit sub-micromolar IC50 potency against PIM3, but with less selectivity for PIM3 versus PIM1 and 2.

Keywords: Proviral Integration site, Maloney murine leukemia virus kinase 3, PIM3, computational screen, diosgenin glycoside, biflavonoid. 


\section{INTRODUCTION}

The PIM family consists of three intracellular serine/threonine kinases that regulate apoptosis, proliferation, migration, and cellular metabolism [1]. PIM kinases share high sequence homology; PIM1 and PIM2 are 53\% identical [2], while PIM1 shares 44 and 71\% identity with PI2 and PIM3, respectively [3]. Overexpression of PIM3 is associated with cancer and inflammation.

In cancer, PIM3 facilitates cell survival through BAD [4]. Unphosphorylated BAD normally binds to $\mathrm{Bcl}-\mathrm{X}(\mathrm{L})$ at the mitochondrial membrane, inhibiting assembly of pro-apoptotic pores in the mitochondrial membrane and enabling apoptosis. Following PIM3-mediated BAD phosphorylation, 14-3-3 binds and releases BAD from the surface of the mitochondria. This leaves $\mathrm{Bcl}-\mathrm{x}(\mathrm{L})$ free to sequester components of the pro-apoptotic pore complex, preventing pore assembly. Mitochondrial membrane integrity is maintained and apoptosis is prevented. Overexpression of PIM3 increases BAD phosphorylation, resulting in more Bcl-X(L) activity and greater resistance to apoptosis. Proto-oncogene PIM3 is overexpressed primarily in tumors of endoderm-derived organs, including the liver, prostate, and pancreatic cancer [5].

PIM3 expression also facilitates inflammation. Tumor Necrosis Factor alpha (TNF- $\alpha$ ) transiently increases Pim-3 mRNA expression in endothelial cells [6]. Endothelial cells participate in the inflammation cascade through the synthesis and secretion of proinflammatory cytokines, including VCAM, ICAM and interleukin. Other studies have reported that PIM kinase activity is required for naïve CD4+ $\mathrm{T}$ cell proliferation and differentiation; inhibiting these kinases in naïve T cells induced a G0/G1 cell cycle arrest [7]. These activities contribute to sustained severity of pathological inflammatory conditions. 
A number of PIM inhibitors are in development. Pan PIM kinase inhibitor SGI-1776, which reduces Bcl-2 anti-apoptotic family member Mcl-1 transcription [8], was tested in clinical trials in non-Hodgkin lymphoma and prostate cancer patients before being withdrawn after possible dose-limiting cardiotoxicity in one patient. After cardiotoxicity from a drug metabolite, analogue TP-3654 has been advanced into clinical trials [9]. A chlorinated 7-azsaindole derivative selectively inhibits PIMs 1 and 3, but not 2, after being derived from rational drug design based on the PIM1 crystal structure, which was used as proxy for PIM3 [10]. Nonselective PIM kinase inhibitor AZD1208 is in phase 1 clinical trials; it decreases proliferation of non-Hodgkin lymphoma cell lines [8] and human prostate cancer xenografts [11]. LGH447 is a non-specific small-molecule inhibitor of PIM kinases; it interrupts G1/S cell cycle transition and affects the expression of different pro-apoptotic proteins like Bcl-2 [8]. M-110 is a highly selective, ATP-competitive inhibitor of PIM kinases with a preference for PIM-3 (IC50=47 nM). M-110 inhibits PIM-1 and PIM-2 with similar IC50s of $2.5 \mu \mathrm{M}$ [12]. Naturally derived molecules have also been reported to target PIM kinases. Quercetagetin [13] and Hispidulin [14] were reported to inhibit PIM1.

Here we use standard drug discovery to identify natural products that are selective PIM3 kinase inhibitors. We employ the SWISS computational modeling tool to generate a predicted PIM3 molecular structure. This predicted structure was used to screen a virtual natural product compound library [15] for small molecules that are predicted to fit the PIM3 ATP binding site using AutoDock Vina. Compound activity was confirmed using biochemical assays of PIM kinase activity. Our results reveal anti-PIM3 activity by 5 of 7 compounds tested. Selectivity and structure-activity relationship of diosgenin glycoside and biflavonoid compounds were explored 
using derivatives. Diosgenin compounds exhibit greater selectivity for PIM3, while biflavonoids exhibit greater potency.

\section{MATERIALS AND METHODS}

\section{In silico docking}

A predicted structure for PIM3 was generated using SWISS-MODEL [16]. Computational docking of library compounds with PIM3 was performed using AutoDock Vina [17]. A region of interest (ROI) containing the ATP binding site within predicted SWISS crystal structure of PIM3 was selected for computational docking. The SWISS program predicted this structure from the PIM1 isoenzyme (2j2i.1.A) [18], which share 71\% homology. The ROI coordinates used were $\mathrm{X}$ center $=31.252, \mathrm{Y}$ center $=-3.074, \mathrm{Z}$ center $=0.0$ and $\mathrm{X}$ size $=18, \mathrm{Y}$ size $=20, \mathrm{Z}$ size $=26$.

\section{Kinase assays and Reagents}

Kinase assays were performed by Reaction Biology under standard conditions. Compounds assessed include PIM3 Kinase Inhibitor M-110 (526526, Millipore, Billerica, MA, USA), dioscin

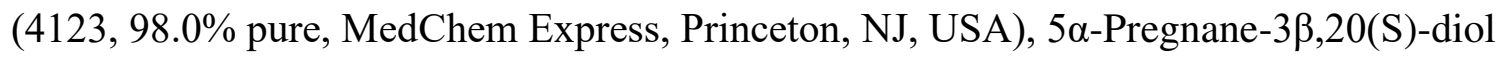
(P705115, 98\% pure, Toronto Research Chemicals, North York, Ontario, Canada), momordicoside L (CFN92307, 98\% pure, Aobious, Gloucester, MA, USA), isorhoifolin (I819700, 98.68\% pure, Toronto Research Chemicals), hinokiflavone (FH74127, Biosynth CarboSynth, Compton, Berkshire, United Kingdom), protodioscin (G0299, 98\% pure Sigma), polyphillin A (A14586, 98\% pure, Adooq Bioscience, Irvine, CA, USA), prosapogenin A (4403, 99\% pure, AvaChem Scientific, San Antonio, TX, USA), timosaponin A3 (1353, 98\% pure, AvaChem Scientific), trillin (1300, 95\% pure, AvaChem Scientific), luteolin 7-rutinoside 
(Carbosynth FL158250, 97\% pure), chelidimerine (Phytopurify BP4054, 98\% pure), 4'-Omethylochnaflavone (Phytopurify BP2020, 95-99\% pure), podocarpus flavone A (Phytopurify BP1816, 95\% pure), luteolin 7-rutinoside (Carbosynth FL158250, 97\% pure), isoginkgetin (Cayman Chem No. 25104, 98\% pure), amentoflavone (Atomax PR-90940, 99\% pure), tetrahydroamentoflavone (ChemScene CS-4945, 98\% pure), taiwanhomoflavone A (ChemFaces CFN96541, 98\% pure), 5',8"-biluteolin (Carbosynth XB161938, 95\% pure). Compounds were prepared in DMSO and diluted to the appropriate concentration.

\section{RESULTS}

\section{Virtual screening a natural products library for putative PIM3 inhibitors}

Since the crystal structure of PIM 3 has not been reported, we used SWISS-MODEL to generate a predicted structure (Fig. 1). SWISS- MODEL is a free online tool which utilizes the primary structure of the protein along with homology modeling to generate the predicted protein structure [16]. We used SWISS-MODEL over other modeling algorithms based on prior success in our hands. PIM3 bears significant sequence homology with PIM1, whose crystal structure has been solved [18]. Not surprisingly, the predicted PIM3 structure is very similar to that reported for PIM1.

Our virtual screening approach was aimed at identifying compounds with a strong fit for the PIM3 ATP binding site, resulting in favorable competition for binding with ATP itself. The ATP binding pocket occurs within residues 46-54 and virtual docking was performed at a threedimensional ROI comprising this site. We first performed docking models using ATP to determine a benchmark for suitable binding affinities for natural products in subsequent studies. 
ATP fits in the PIM3 binding site with a computationally determined energy of $-8.0 \mathrm{kcal} / \mathrm{mol}$ (Fig. 1). Selective PIM3 inhibitor M-110 binds the predicted PIM3 structure in silico with a predicted binding energy of $-10.2 \mathrm{kcal} / \mathrm{mol}$.

We then conducted a virtual docking screen against the same ROI using our virtual natural products library. This library, which we describe elsewhere [15], comprises 98,000 unique and diverse naturally-derived molecules assembled from smaller libraries [19-23]. AutoDock Vina returned 2,028 molecules, or $2.07 \%$ of the screened library, that are predicted to fit in the modeled PIM3 binding site with affinities less than $-8.0 \mathrm{kcal} / \mathrm{mol}$. An analysis of structural similarity among the 100 molecules with the highest binding scores reveals enrichment for glycoside flavone, bianthrone, steroidal diosgenin glycoside, biflavonoid, and alkaloid derivatives.

\section{Validating PIM3 kinase activity}

We sought to validate PIM3 kinase inhibition in a small panel of structurally diverse molecules representing different groups of structurally similar scaffolds identified in the computational screen (Fig. 2) using biochemical assays with purified PIM3. Only momordicoside L showed no PIM3 inhibition at any concentration. All other molecules tested showed inhibition of PIM3 at the highest concentration, though only with $38.6 \%$ inhibition for chelidimerine. Calculation of IC50 values resulted in a range that reached as low as $2.17 \mu \mathrm{M}$ for hinokiflavone (Fig. 3, Table 1). The results from the biochemical assay demonstrate the successful querying of a natural products library by computational docking screening against a kinase, even when the structure is generated by in silico modeling. 


\section{Selective PIM3 inhibition by diosgenin glycoside analogs}

We next sought to probe the anti-PIM3 pharmacophore of dioscin by assessing PIM3 inhibition with five structurally similar saponin molecules. We measured PIM3 inhibition by a range of concentrations of protodioscin, polyphillin $\mathrm{A}$, timosaponin $\mathrm{A} 3$, prosapogenin $\mathrm{A}$, and trillin (Fig. 4). Timosaponin A3 inhibits PIM3 kinase activity with a calculated IC50 of $21.8 \mu \mathrm{M}$, meaning it is twice as potent as dioscin. Polyphillin A and Trillin inhibit PIM3 kinase with calculated IC50 values of 60.3 and $73.4 \mu \mathrm{M}$, respectively (Fig. 5, Table 1). Both of these compounds are roughly half as potent as dioscin. Meanwhile prosapogenin A and protodioscin bear minimal activity against PIM3.

We then sought to examine the selectivity of these compounds for PIM3 versus PIM1 and 2, particularly since there is high sequence homology with PIM1 and since the structure of PIM3 was computationally predicted based on that of PIM1. Dioscin, polyphyllin A, and timosaponin A3 were tested against PIM1 and PIM2 at a range of concentrations (Fig. 6, Table 1). Dioscin proved the most selective saponin analog for PIM3, demonstrating negligible inhibition of either PIM1 or PIM2. Timosaponin A3 and polyphillin A are less selective for PIM3. Timosaponin A3 inhibits PIM3 with an IC50 of 20.89uM compared to 142.9 and 92.45uM for PIM 1 and 2, respectively, while polyphillin A inhibits PIM3 with an IC50 of 60.05um versus 113.1uM for PIM1 and limited inhibition of PIM2. Despite significant changes in molecular structure among the six analogs tested, the change in PIM 3 activity and selectivity is subtle. It appears that modifications in the steroid structure or its attached carbohydrates can both alter activity. 


\section{Potency and selectivity of the biflavonoid scaffold}

In parallel, we also sought to better define the structure of the anti-PIM3 pharmacophore in hinokiflavone. We selected structurally similar molecules amentoflavone, podocarpus flavone A, taiwanhomoflavone A, tetrahydroamentoflavone, 4'-O-methylochnaflavone, isogingketin, and 5',8' biluteolin compounds for assessment in PIM3 kinase assays (Fig. 7). Among these compounds, amentoflavone showed the greatest inhibition of PIM3 kinase with a calculated IC50 of 178.3nM, roughly an order of magnitude more potent that hinokiflavone (Fig. 8, table 1). Podocarpus flavone A and 5',8'-biluteolin also inhibit PIM3 with potencies greater than that of hinokiflavone (IC50 values of 755.9nM and 491.6nM, respectively). Meanwhile, 4'-Omethylochnaflavone and tetrahydroamentoflavone inhibit PIM3 with potencies several times higher than hinokiflavone (IC50 values of 11.84uM and 13.30uM, respectively). Isoginkgetin and taiwanhomoflavone A inhibit PIM3 only at the highest concentrations tested.

We tested the selectivity of the most potent biflavonoids. Amentoflavone, podocarpus flavone A, 5',8" biluteolin, were tested for inhibition against PIM1 and PIM2 kinases at a range of concentrations. Selectivity was limited with respect to PIM kinases 1 and 3 (Fig. 9, Table 1). Amentoflavone inhibits PIM1 with an IC50 of 296nM, less than double the potency against PIM3 inhibition. Podocarpus Flavone A is more potent against PIM1 than PIM3, inhibiting PIM3 with an IC50 of 417.1nM (Fig. 9, table 1). Inhibition of PIM2 is much reduced in these scaffolds. The calculated IC50s versus PIM2 are 7.65 and $42.72 \mathrm{uM}$ for amentoflavone and podocarpus flavone A, respectively. 


\section{DISCUSSION}

Computational screening successfully revealed natural product inhibitors of PIM3, despite the use of a computationally derived protein structure. We explored selectivity and potency more fully among diosgenin glycoside relatives of dioscin and biflavonoid analogs of hinokiflavone. Diosgenin glycosides, among which timosaponin A3 is the most potent, are more selective against PIM3 than biflavonoids. Meanwhile, biflavonoids are more potent.

Dioscin and biflavonoids have been widely studied in cell models. Both classes of compounds and derivatives of each assessed here have shown activity in blocking cellular activities and pathways associated with inflammation and caner progression, both of which are consistent with PIM kinase inhibition. While the number of reports is so great to preclude a thorough review here, both classes of compounds display similar biological effects that suggest that they share some common mechanism. Both classes of compounds have been shown to arrest cell growth at G2/M in a wide range of cancer cell lines, where they induce apoptosis with ROS signaling and mitochondrial involvement $[24,25]$. Cancer cell apoptosis induced by both compounds is via altered expression of proapoptotic and antiapoptotic mitochondrial proteins like Bax and Bcl-2, as well as caspase proteases [26, 27]. In the context of cell growth inhibition, both compound classes are linked to cellular signaling consistent with PIM kinase targeting, particularly in the case of $\mathrm{NF}-\kappa \mathrm{B}$ signaling [28, 29]. And other pathway-level effects observed in growth arrest are also shared by both compound classes, an example of which is that both inhibit skp2-mediated ubiquitylation [30,31]. Beyond effects on cell growth, both classes of compounds also suppress glycolysis [30, 32] and modulate autophagy [33, 34]. Both classes of compounds have been reported to sensitize cancer cells to other anticancer agents [28, 35]. Further, both compounds block cancer cell migration, invasion, and TGF $\beta$-induced epithelial-mesenchymal 
transition $[36,37]$. Cleary these very distinct classes of compounds share one or more molecular targets. That we identify PIM kinases are a common target for both classes of compounds supports that this mechanism is physiologically relevant.

The biological activity of naturally occurring compounds is first revealed from use at the system level, especially in cases where such compounds are used in traditional medicine, or in phenotypic assays. Effects on cellular pathways and gene transcription can provide more detail that can elucidate mechanism of action. Often, however, identifying a precise molecular target is challenging. A case in point is the lack of a defined molecular target for dioscin. Conversely, a number of other molecular targets have been previously reported for amentoflavone, including tryptase [15], fatty acid synthase [38], PARP-1 [39], cathepsin B [40], and cyclooxygenases [41]. Some of these molecular targets could explain the biological activities reported at the cell and pathway level. Further, biochemical activity against UDP-glucoronosyltransferases [42] and cytochrome p450 (CYP) enzymes [43] reveal potential toxic liabilities. In fact, the severally reported biochemical activities of amentoflavone demonstrate how natural products are likely to have multiple molecular targets or, as may be the case for amentoflavone, may at as pan-assay inhibitors.

Natural products are important sources of new therapeutics, but often display complex biology. Defining specific molecular mechanisms of action, including their potency and selectivity, can provide important understanding of required to build a meaningful rationale for clinical development. Efforts to identify natural products with activity against the clinicallyrelevant target PIM3 reported here could explain previously reported cellular pharmacology of diosgenin glycosides and biflavonoids. 


\section{Acknowledgments}

Funding for the research was provided by the Department of Physiology and Developmental Biology at the College of Life Sciences at Brigham Young University, Provo.

\section{Conflicts of Interest}

The authors declare that they have no conflict of interest. 


\section{REFERENCES}

1. Koblish, H., et al., Preclinical characterization of INCB053914, a novel pan-PIM kinase inhibitor, alone and in combination with anticancer agents, in models of hematologic malignancies. PLoS One, 2018. 13(6): p. e0199108.

2. Brault, L., et al., PIM serine/threonine kinases in the pathogenesis and therapy of hematologic malignancies and solid cancers. Haematologica, 2010. 95(6): p. 1004-15.

3. Li, Y.Y. and N. Mukaida, Pathophysiological roles of Pim-3 kinase in pancreatic cancer development and progression. World J Gastroenterol, 2014. 20(28): p. 9392-404.

4. Li, Y.Y., et al., Pim-3, a proto-oncogene with serine/threonine kinase activity, is aberrantly expressed in human pancreatic cancer and phosphorylates bad to block badmediated apoptosis in human pancreatic cancer cell lines. Cancer Res, 2006. 66(13): p. 6741-7.

5. Blanco-Aparicio, C. and A. Carnero, Pim kinases in cancer: diagnostic, prognostic and treatment opportunities. Biochem Pharmacol, 2013. 85(5): p. 629-643.

6. Yang, $\mathrm{H}$., et al., Pim protein kinase-3 is regulated by TNF- $\alpha$ and promotes endothelial cell sprouting. Molecules and cells, 2011. 32(3): p. 235-241.

7. Jackson, L.J., et al., The role of PIM kinases in human and mouse CD4+ T cell activation and inflammatory bowel disease. Cell Immunol, 2012. 272(2): p. 200-13.

8. Zhang, X., et al., PIM Kinase as an Executional Target in Cancer. J Cancer Prev, 2018. 23(3): p. 109-116.

9. Foulks, J.M., et al., A small-molecule inhibitor of PIM kinases as a potential treatment for urothelial carcinomas. Neoplasia, 2014. 16(5): p. 403-12.

10. Nakano, H., et al., Design and Synthesis of Potent and Selective PIM Kinase Inhibitors by Targeting Unique Structure of ATP-Binding Pocket. ACS Med Chem Lett, 2017. 8(5): p. 504-509.

11. Kirschner, A.N., et al., PIM kinase inhibitor AZD1208 for treatment of MYC-driven prostate cancer. J Natl Cancer Inst, 2015. 107(2).

12. Chang, M., et al., PIM kinase inhibitors downregulate STAT3(Tyr705) phosphorylation. Mol Cancer Ther, 2010. 9(9): p. 2478-87.

13. Jie, W., et al., Inhibition of Pim-1 attenuates the proliferation and migration in nasopharyngeal carcinoma cells. Asian Pac J Trop Med, 2012. 5(8): p. 645-50.

14. Liu, K., et al., Hispidulin suppresses cell growth and metastasis by targeting PIM1 through JAK2/STAT3 signaling in colorectal cancer. Cancer Sci, 2018. 109(5): p. 13691381.

15. Fazio, N.F., et al., A natural product biflavonoid scaffold with anti-tryptase activity. Naunyn Schmiedebergs Arch Pharmacol, 2021. 394(1): p. 107-115.

16. Waterhouse, A., et al., SWISS-MODEL: homology modelling of protein structures and complexes. Nucleic Acids Res, 2018. 46(W1): p. W296-W303.

17. Morris, G.M., et al., AutoDock4 and AutoDockTools4: Automated docking with selective receptor flexibility. J Comput Chem, 2009. 30(16): p. 2785-91.

18. Fedorov, O., et al., A systematic interaction map of validated kinase inhibitors with Ser/Thr kinases. Proc Natl Acad Sci U S A, 2007. 104(51): p. 20523-8.

19. Ntie-Kang, F., et al., AfroDb: a select highly potent and diverse natural product library from African medicinal plants. PLoS One, 2013. 8(10): p. e78085.

20. $\mathrm{Ru}$, J., et al., TCMSP: a database of systems pharmacology for drug discovery from herbal medicines. J Cheminform, 2014. 6: p. 13.

21. Ntie-Kang, F., et al., NANPDB: A Resource for Natural Products from Northern African Sources. J Nat Prod, 2017. 80(7): p. 2067-2076.

22. Chemicals, I. Indofine Natural Products. 2016; Available from: https://zinc.docking.org/catalogs/indofinenp/. 
23. Introbioscreen. IBScreen. 2018; Available from: http://zinc.docking.org/catalogs/ibs/.

24. Zhao, X., et al., Dioscin Induces Apoptosis in Human Cervical Carcinoma HeLa and SiHa Cells through ROS-Mediated DNA Damage and the Mitochondrial Signaling Pathway. Molecules, 2016. 21(6).

25. Pei, J.S., et al., Amentoflavone induces cell-cycle arrest and apoptosis in MCF-7 human breast cancer cells via mitochondria-dependent pathway. In Vivo, 2012. 26(6): p. 96370.

26. Zhang, G., et al., Dioscin suppresses hepatocellular carcinoma tumor growth by inducing apoptosis and regulation of TP53, BAX, BCL2 and cleaved CASP3. Phytomedicine, 2016. 23(12): p. 1329-1336.

27. Guruvayoorappan, C. and G. Kuttan, Amentoflavone stimulates apoptosis in B16F-10 melanoma cells by regulating bcl-2, p53 as well as caspase-3 genes and regulates the nitric oxide as well as proinflammatory cytokine production in B16F-10 melanoma cells, tumor associated macrophages and peritoneal macrophages. J Exp Ther Oncol, 2008. 7(3): p. 207-18.

28. Wang, C., et al., Dioscin strengthens the efficiency of adriamycin in MCF-7 and MCF7/ADR cells through autophagy induction: More than just down-regulation of MDR1. Sci Rep, 2016. 6: p. 28403.

29. Lee, Y.J., et al., Suppression of ERK/NF-KB Activation Is Associated With Amentoflavone-Inhibited Osteosarcoma Progression. Anticancer Res, 2019. 39(7): p. 3669-3675.

30. Zhou, L., et al., Cdh1-mediated Skp2 degradation by dioscin reprogrammes aerobic glycolysis and inhibits colorectal cancer cells growth. EBioMedicine, 2020. 51: p. 102570.

31. Liu, H., Q. Yue, and S. He, Amentoflavone suppresses tumor growth in ovarian cancer by modulating Skp2. Life Sci, 2017. 189: p. 96-105.

32. Liu, B. and S. Yu, Amentoflavone suppresses hepatocellular carcinoma by repressing hexokinase 2 expression through inhibiting JAK2/STAT3 signaling. Biomed Pharmacother, 2018. 107: p. 243-253.

33. Mao, Z., et al., Potent effects of dioscin against hepatocellular carcinoma through regulating TP53-induced glycolysis and apoptosis regulator (TIGAR)-mediated apoptosis, autophagy, and DNA damage. Br J Pharmacol, 2019. 176(7): p. 919-937.

34. Chen, Y., et al., Amentoflavone suppresses cell proliferation and induces cell death through triggering autophagy-dependent ferroptosis in human glioma. Life Sci, 2020. 247: p. 117425.

35. Chen, C.H., et al., Anticancer Efficacy and Mechanism of Amentoflavone for Sensitizing Oral Squamous Cell Carcinoma to Cisplatin. Anticancer Res, 2020. 40(12): p. 67236732.

36. Lim, W.C., et al., Dioscin suppresses TGF- $\beta 1$-induced epithelial-mesenchymal transition and suppresses A549 lung cancer migration and invasion. Bioorg Med Chem Lett, 2017. 27(15): p. 3342-3348.

37. Kim, G.L., et al., Amentoflavone, active compound of Selaginella tamariscina, inhibits in vitro and in vivo TGF- $\beta$-induced metastasis of human cancer cells. Arch Biochem Biophys, 2020. 687: p. 108384.

38. Lee, J.S., et al., Fatty acid synthase inhibition by amentoflavone induces apoptosis and antiproliferation in human breast cancer cells. Biol Pharm Bull, 2009. 32(8): p. 1427-32.

39. Hu, X.L., et al., Identification of amentoflavone as a potent highly selective PARP-1 inhibitor and its potentiation on carboplatin in human non-small cell lung cancer. Phytomedicine, 2018. 50: p. 88-98.

40. Pan, X., et al., Amentoflavone and its derivatives as novel natural inhibitors of human Cathepsin B. Bioorg Med Chem, 2005. 13(20): p. 5819-25. 
41. Seaver, B. and J.R. Smith, Inhibition of COX isoforms by nutraceuticals. J Herb Pharmacother, 2004. 4(2): p. 11-8.

42. Lv, X., et al., Amentoflavone is a potent broad-spectrum inhibitor of human UDPglucuronosyltransferases. Chem Biol Interact, 2018. 284: p. 48-55.

43. Park, S.Y., et al., Strong and Selective Inhibitory Effects of the Biflavonoid Selamariscina A against CYP2C8 and CYP2C9 Enzyme Activities in Human Liver Microsomes.

Pharmaceutics, 2020. 12(4). 


\section{FIGURE LEGENDS}
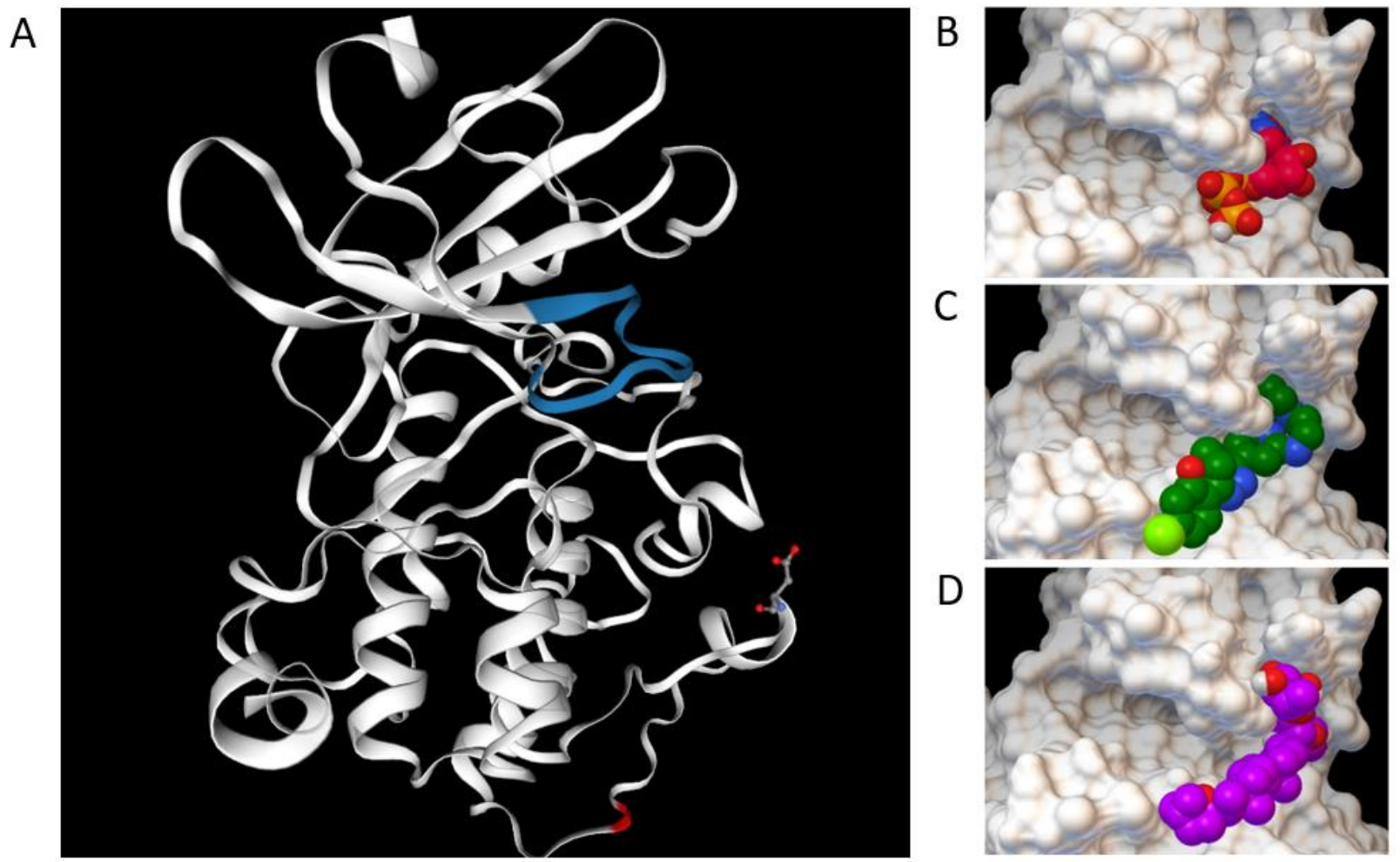

Fig 1. Predicted SWISS crystal structure of PIM3.

A. The entire protein with the ATP binding pocket labeled blue. B-D. The ATP binding pocket of the predicted PIM3 structure fitted to ATP (B), PIM kinase inhibitor M-110 (C), and Dioscin (D). 


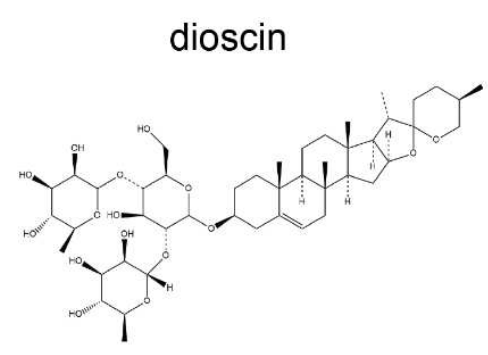

hinokiflavone

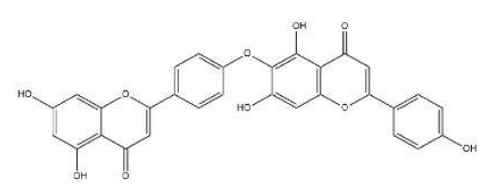

5-a-pregnane

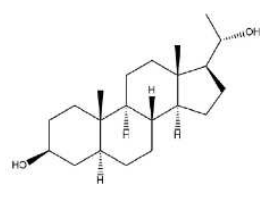

luteolin 7-rutinoside momordicoside L

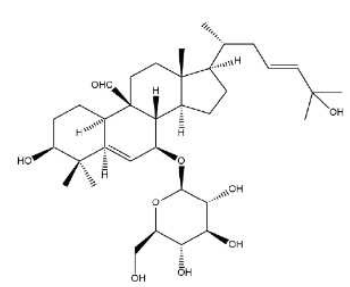

chelidimerine
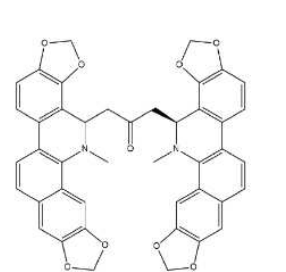

Fig 2. Compounds selected for validation from virtual screening.
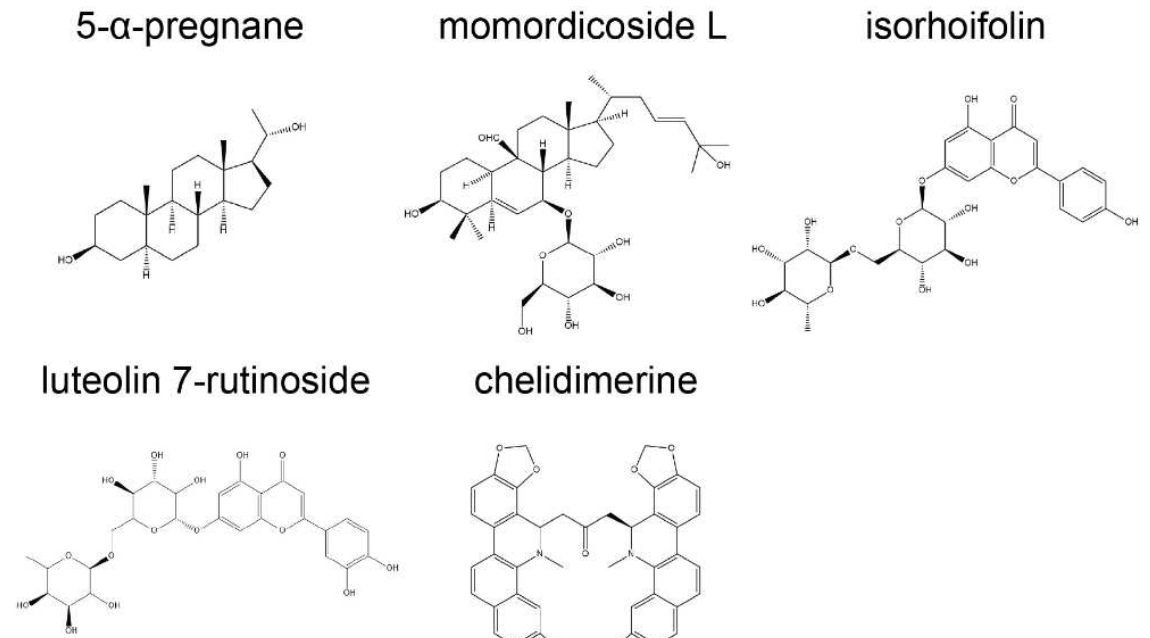


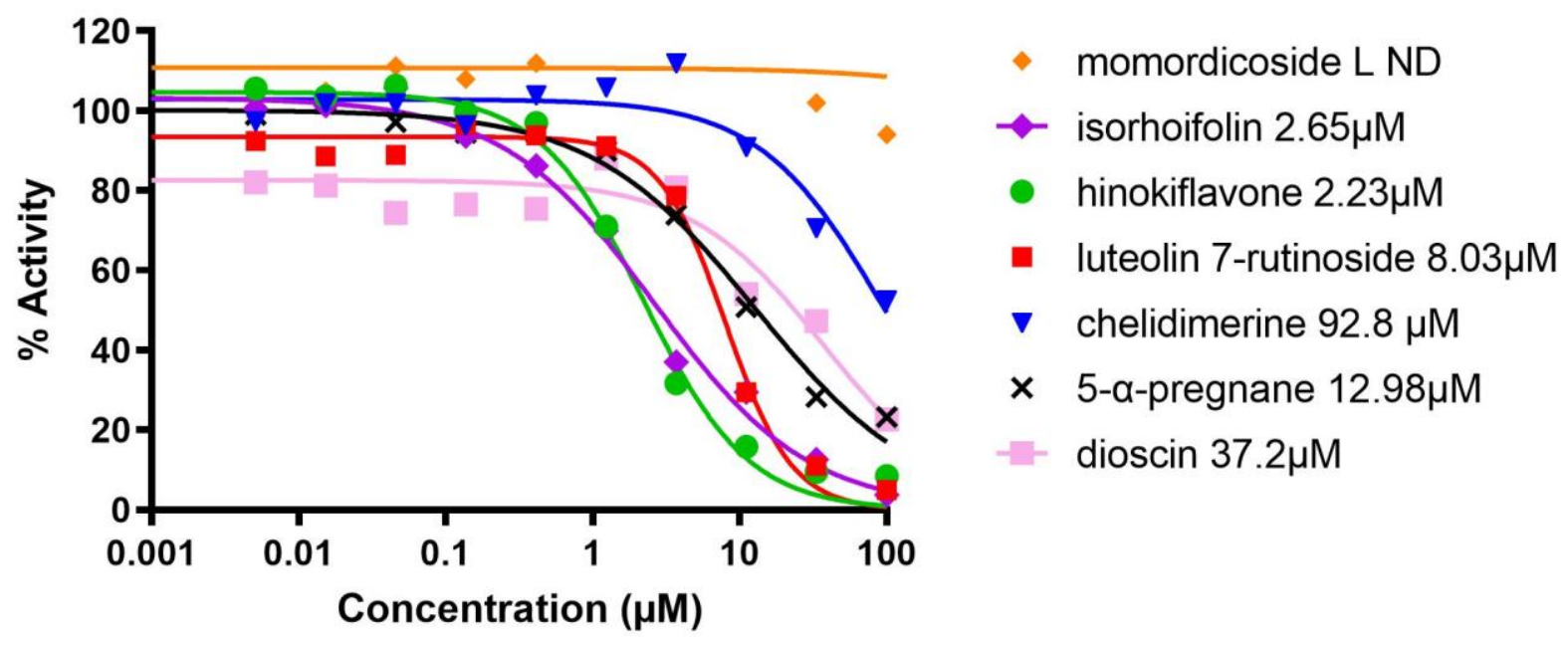

Fig 3. Validation of computationally predicted PIM3 kinase inhibition.

PIM3 activity at a range of concentration of the indicated, with fitted curves and calculated IC50 values. $\mathrm{ND}=$ not determined. 

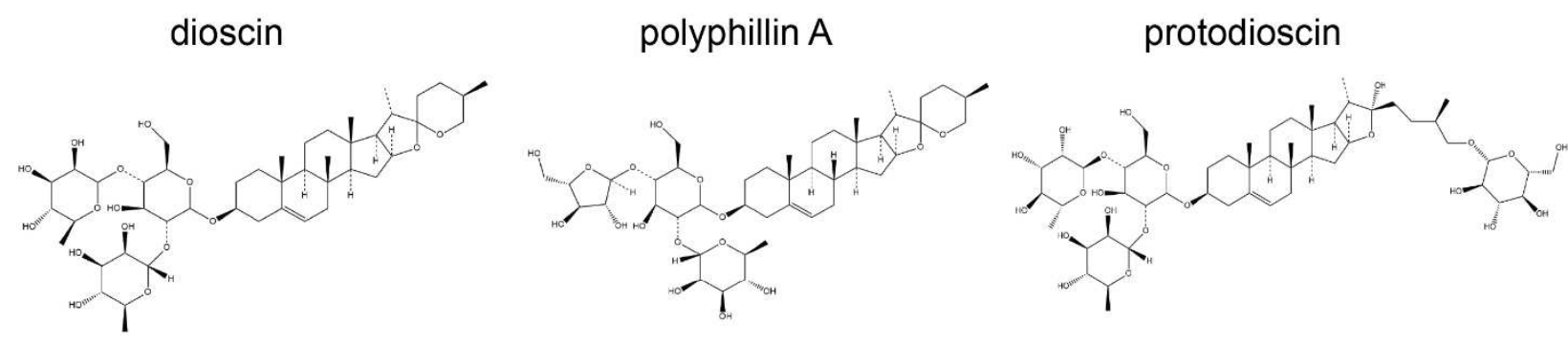

prosapogenin A

timosaponin A3

trillin
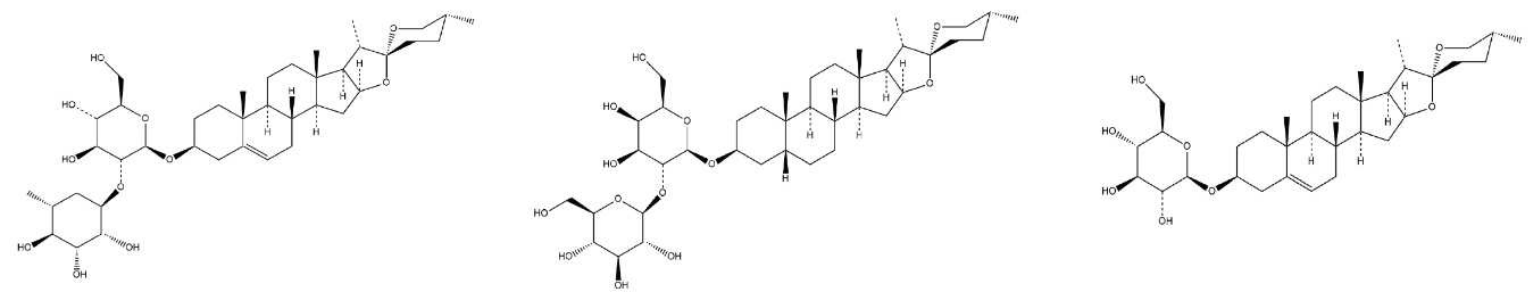

Fig 4. Diosgenin glycoside analogs. 


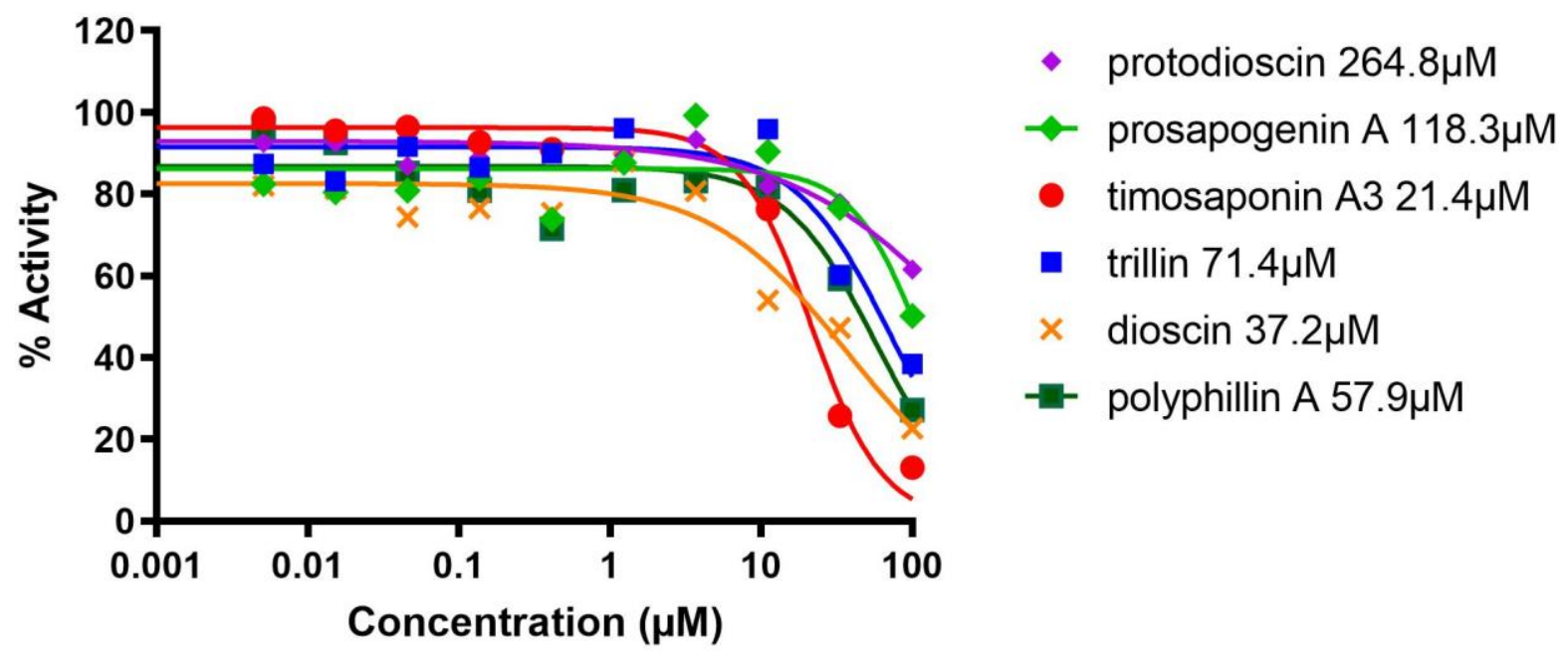

Fig 5. PIM3 kinase inhibition by diosgenin glycosides.

PIM3 activity at a range of concentration of the indicated, with fitted curves and calculated IC50 values. 

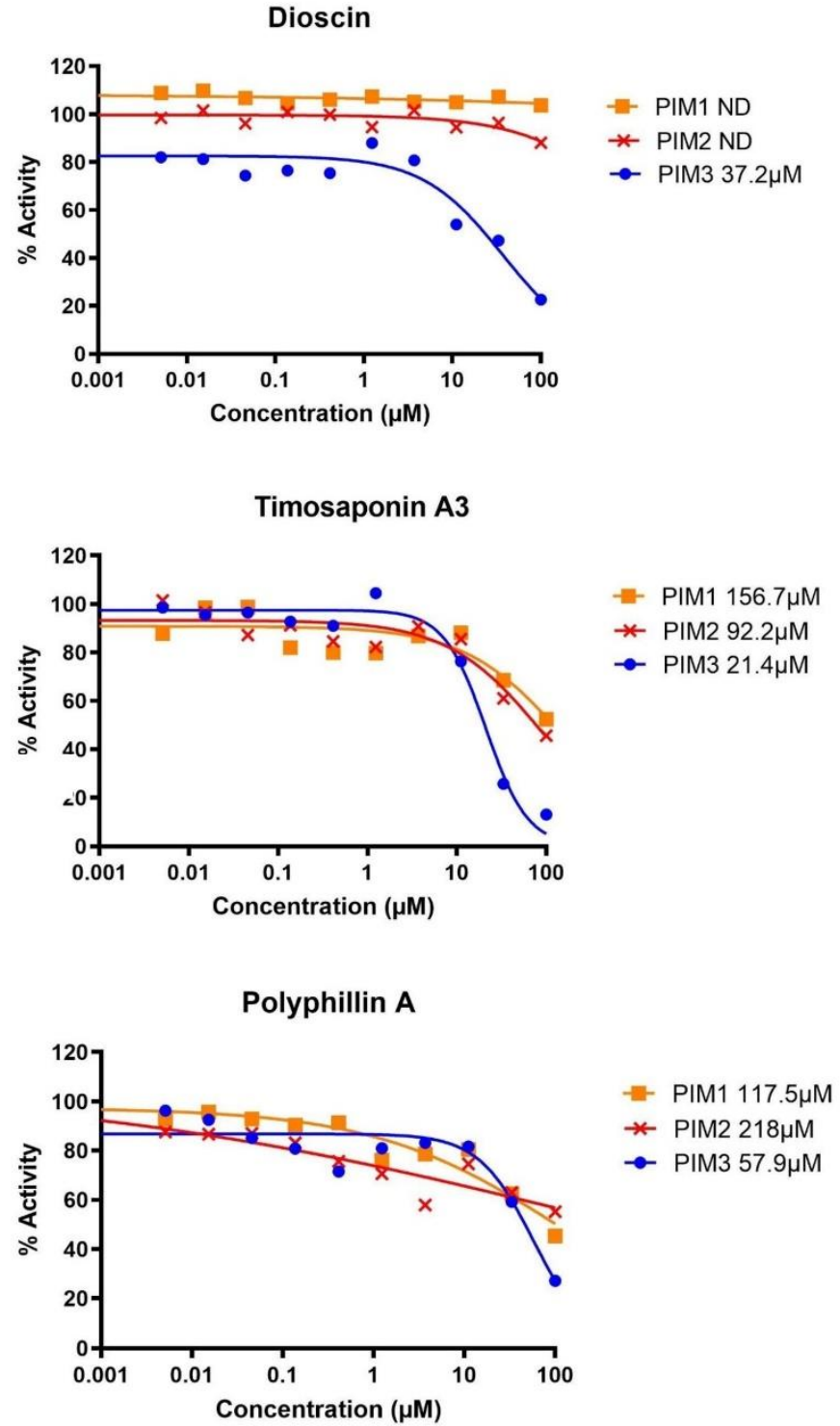

Fig 6. PIM kinase selectivity of diosgenin glycoside analogs.

PIM1, 2, and 3 activity in the presence of a range of compound concentrations, with fitted curves and calculated IC50 values. ND= not determinable from the curve. 

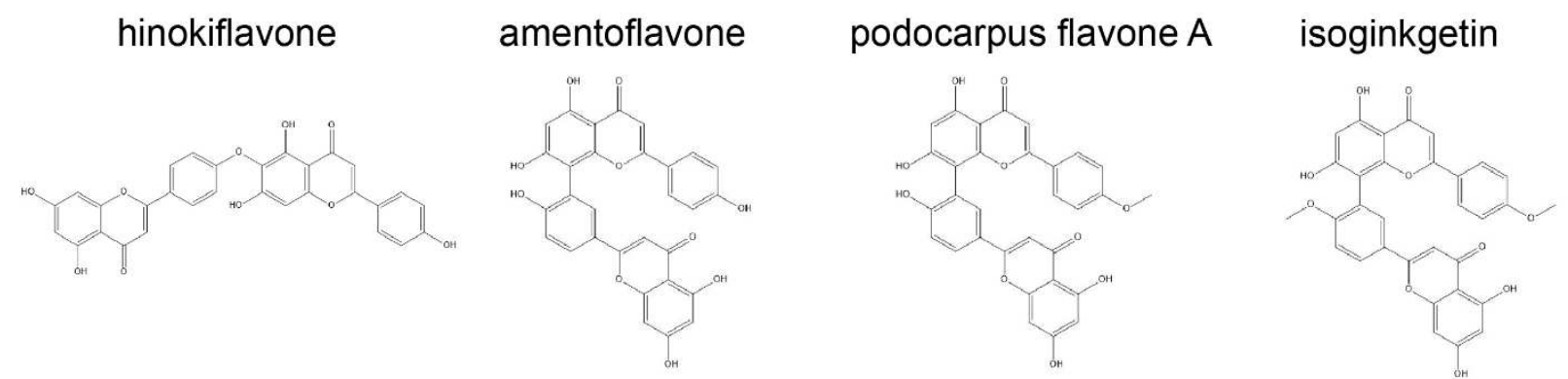

taiwanhomoflavone A 4'-O-methylochnaflavone
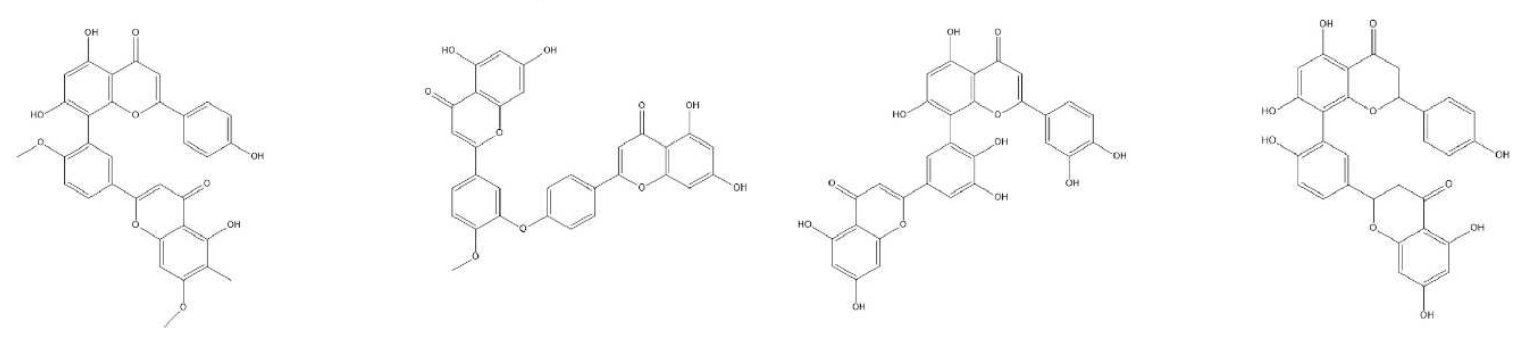

Fig 7. Biflavonoid analogs. 


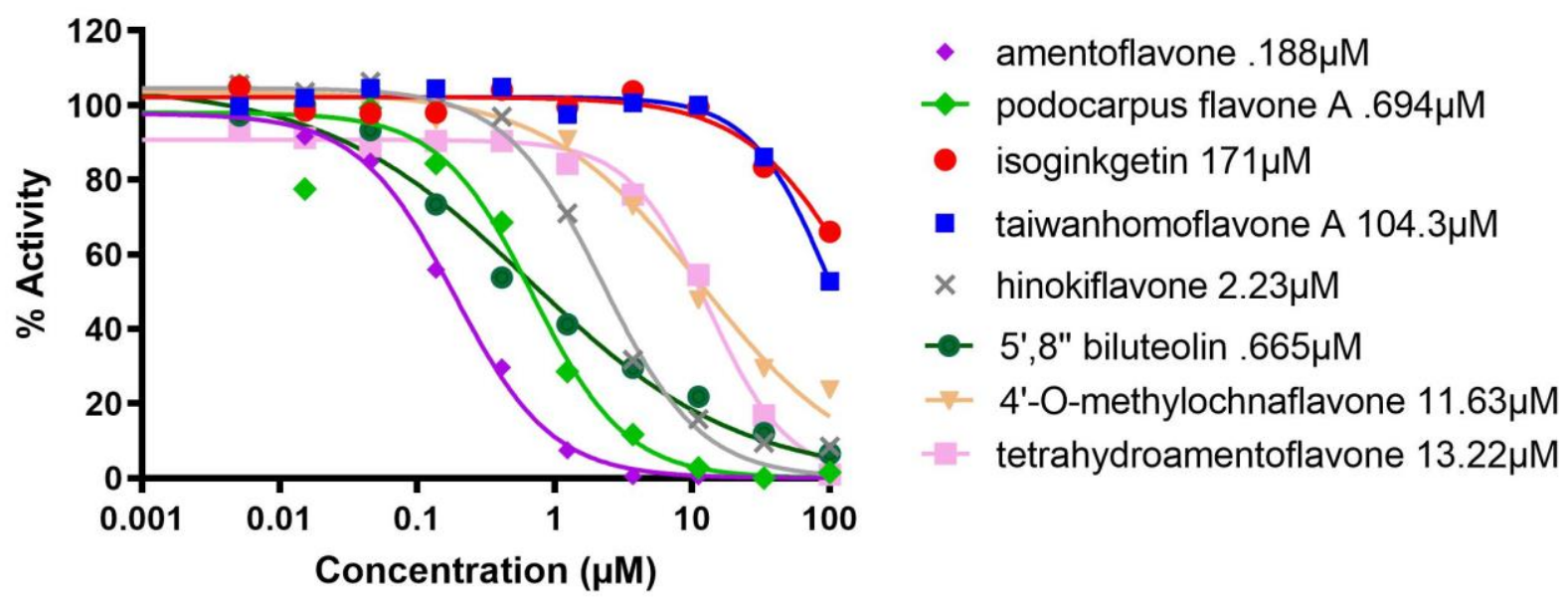

Fig 8. PIM3 inhibition by biflavonoids.

PIM3 activity at a range of concentration of the indicated, with fitted curves and calculated IC50 values. 

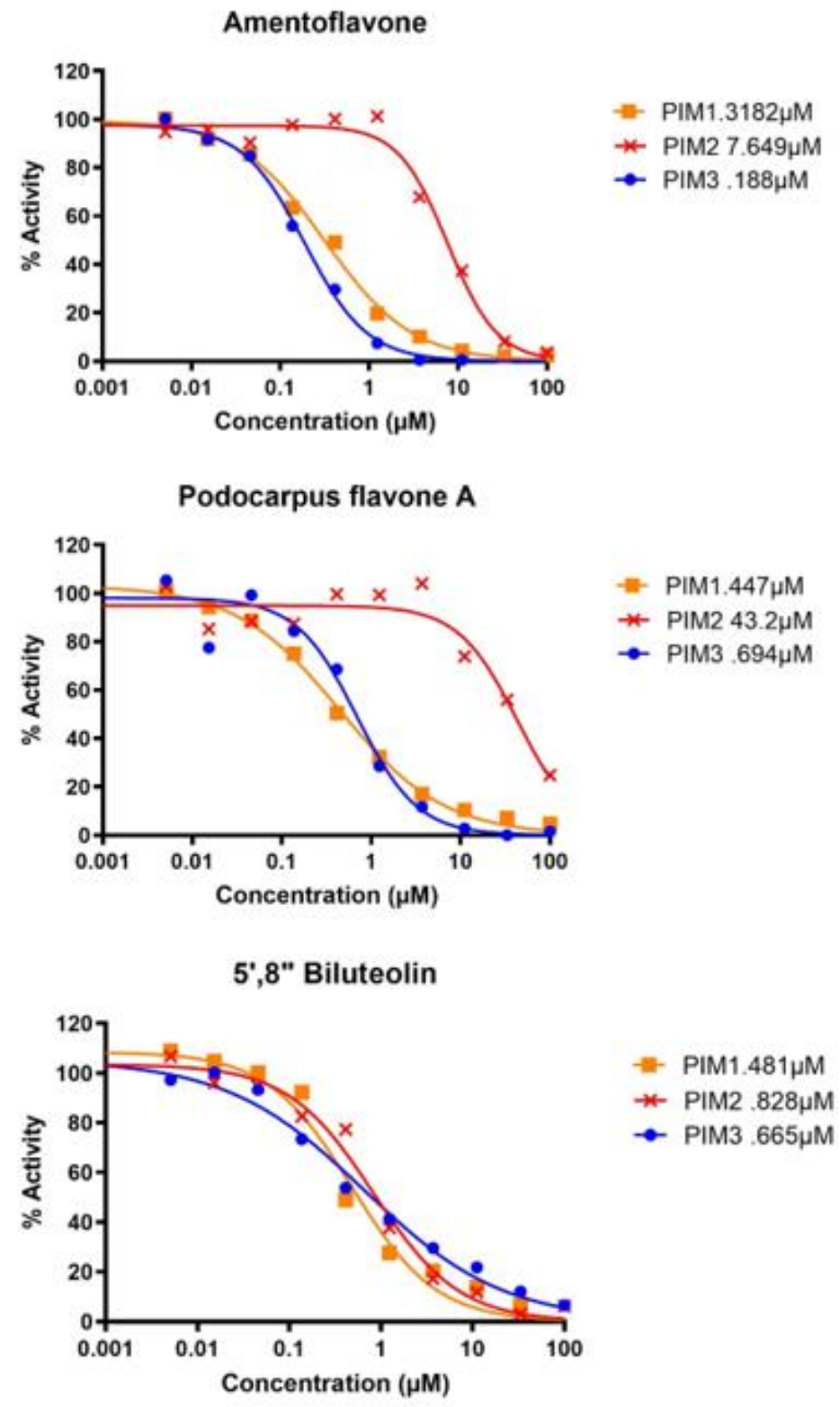

Fig 9. PIM kinase selectivity of biflavonoids.

PIM1, 2, and 3 activity in the presence of a range of compound concentrations, with fitted curves and calculated IC50 values. 


\begin{tabular}{lccc} 
Compound & PIM1 & PIM2 & PIM3 \\
\hline 5- $\alpha$-pregnane & & & $12.98 \mu \mathrm{M}$ \\
momordicoside L & & $\mathrm{ND}$ \\
isorhoifolin & $22.9 \mu \mathrm{M}$ & $\mathrm{ND}$ & $2.65 \mu \mathrm{M}$ \\
luteolin 7-rutinoside & & & $8.03 \mu \mathrm{M}$ \\
chelidimerine & & & $92.8 \mu \mathrm{M}$ \\
\hline dioscin & $\mathrm{ND}$ & $\mathrm{ND}$ & $37.2 \mu \mathrm{M}$ \\
polyphillin A & $117.5 \mu \mathrm{M}$ & $218.0 \mu \mathrm{M}$ & $57.9 \mu \mathrm{M}$ \\
protodioscin & & & $264.8 \mu \mathrm{M}$ \\
prosapogenin A & & & $118.3 \mu \mathrm{M}$ \\
timosaponin A3 & $156.7 \mu \mathrm{M}$ & $92.2 \mu \mathrm{M}$ & $21.4 \mu \mathrm{M}$ \\
trillin & & & $71.4 \mu \mathrm{M}$ \\
\hline hinokiflavone & & & $2.23 \mu \mathrm{M}$ \\
amentoflavone & & & $.188 \mu \mathrm{M}$ \\
podocarpus flavone A & $.447 \mu \mathrm{M}$ & $43.2 \mu \mathrm{M}$ & $.694 \mu \mathrm{M}$ \\
isoginkgetin & & & $171 \mu \mathrm{M}$ \\
taiwanhomoflavone A & & & $104.3 \mu \mathrm{M}$ \\
4'-O-methylochnaflavone & & & $11.63 \mu \mathrm{M}$ \\
5',8" biluteolin & $.481 \mu \mathrm{M}$ & $.828 \mu \mathrm{M}$ & $.665 \mu \mathrm{M}$ \\
tetrahydroamentoflavone & & & $13.22 \mu \mathrm{M}$
\end{tabular}

Table 1. IC50 values against PIM kinases. 


\section{Figures}
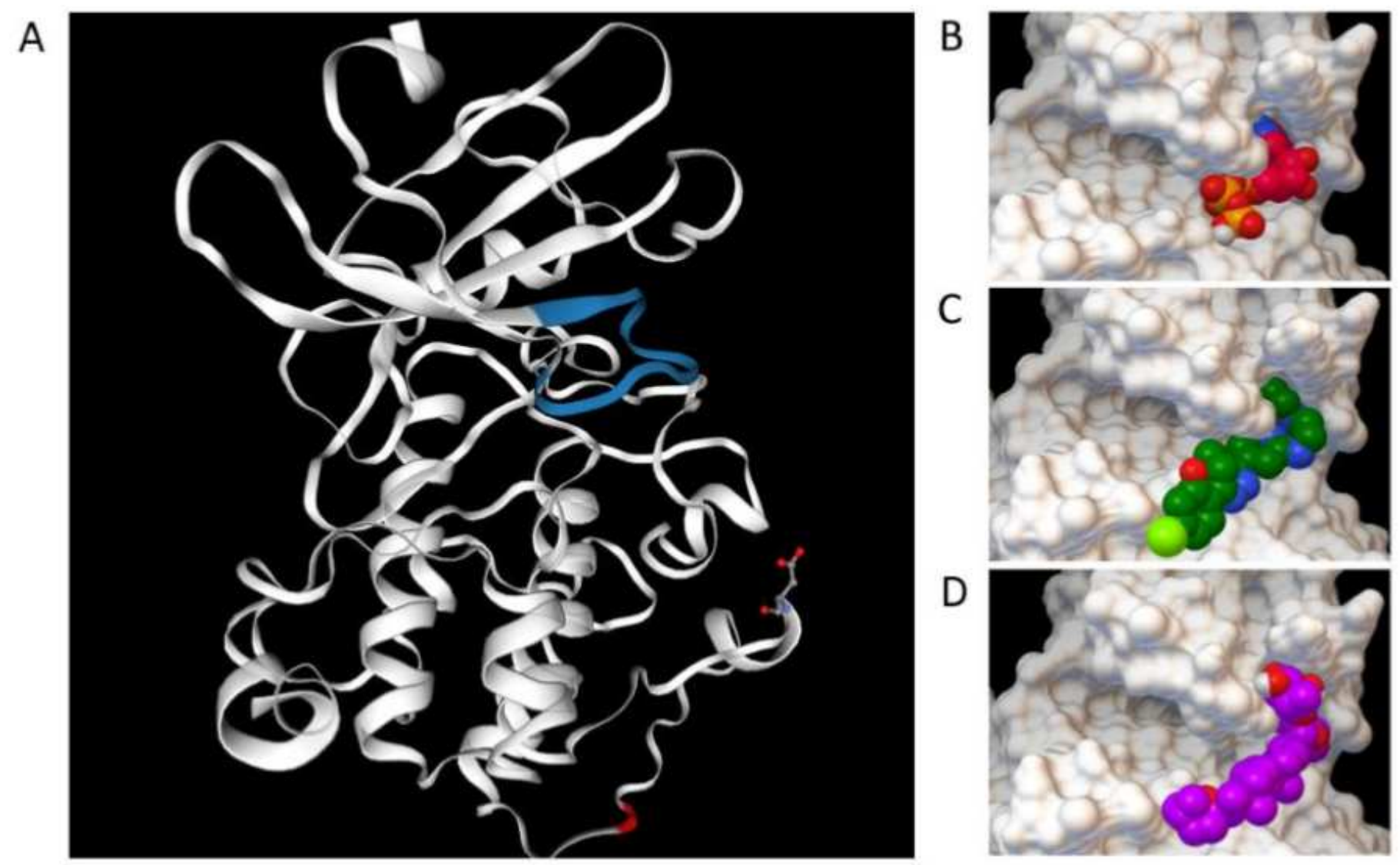

\section{Figure 1}

Predicted SWISS crystal structure of PIM3. A. The entire protein with the ATP binding pocket labeled blue. B-D. The ATP binding pocket of the predicted PIM3 structure fitted to ATP (B), PIM kinase inhibitor M-110 (C), and Dioscin (D).

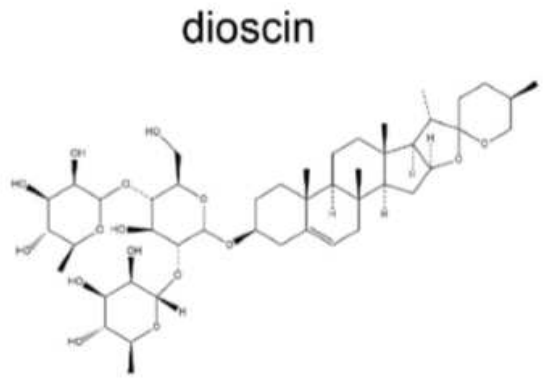

hinokiflavone

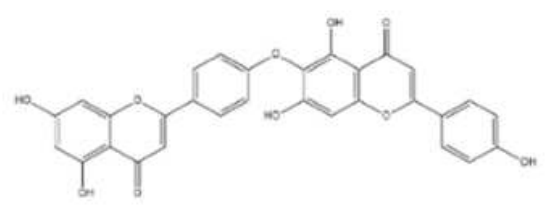

5-a-pregnane

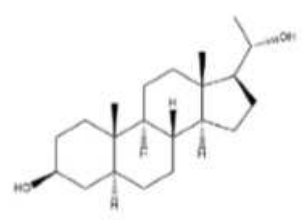

luteolin 7-rutinoside

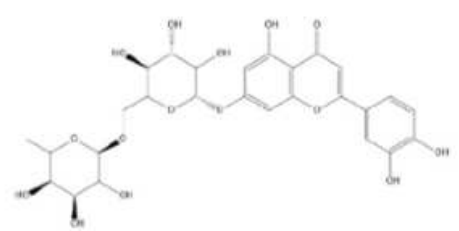

momordicoside L
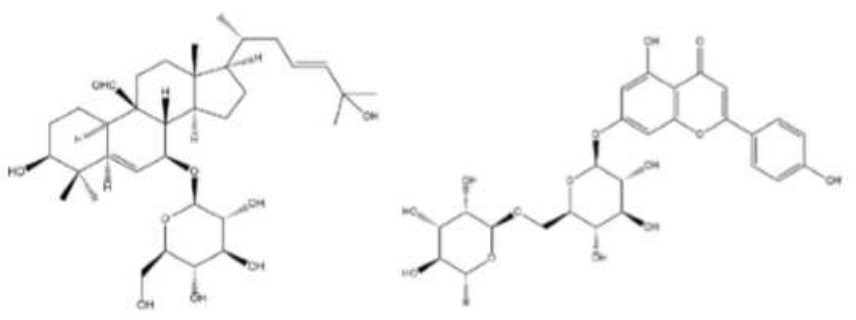

chelidimerine

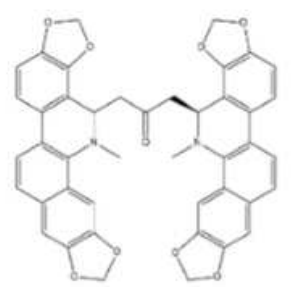


Figure 2

Compounds selected for validation from virtual screening.

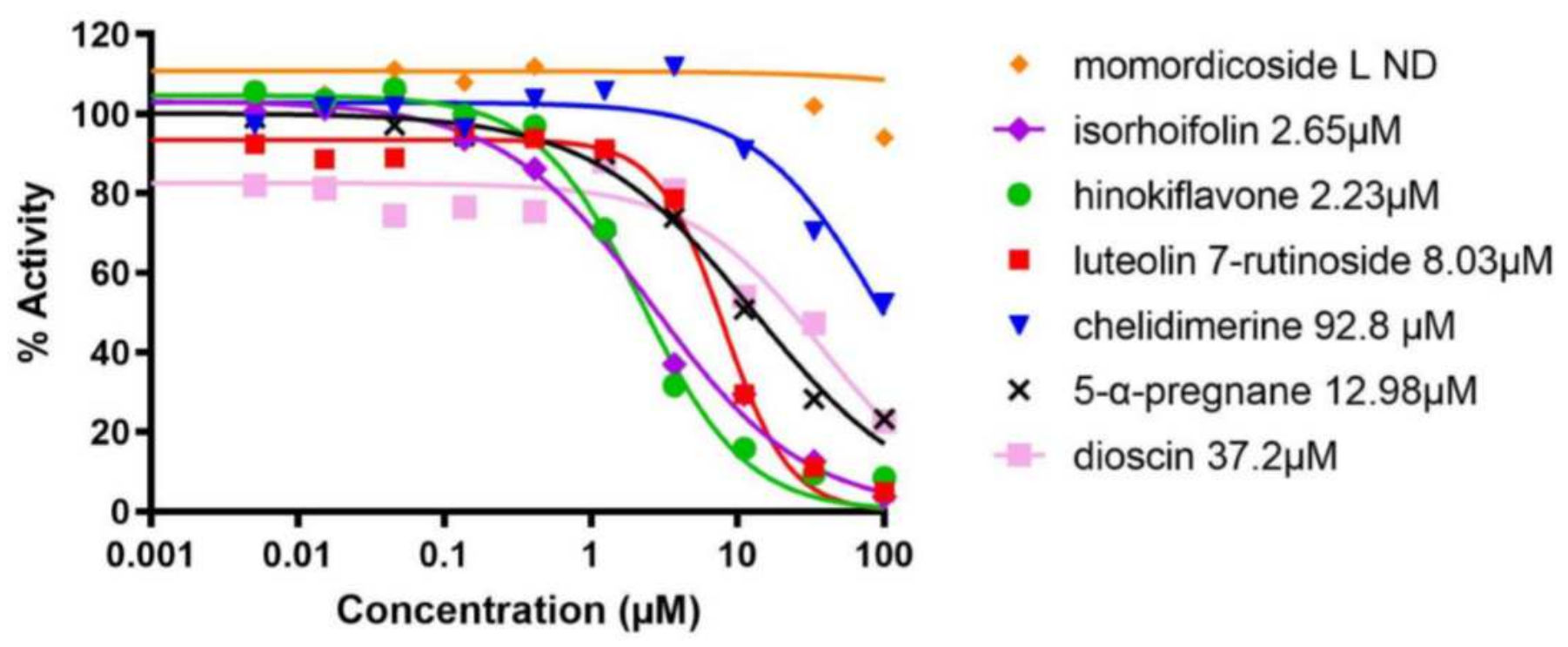

Figure 3

Validation of computationally predicted PIM3 kinase inhibition. PIM3 activity at a range of concentration of the indicated, with fitted curves and calculated IC50 values. ND= not determined.

dioscin

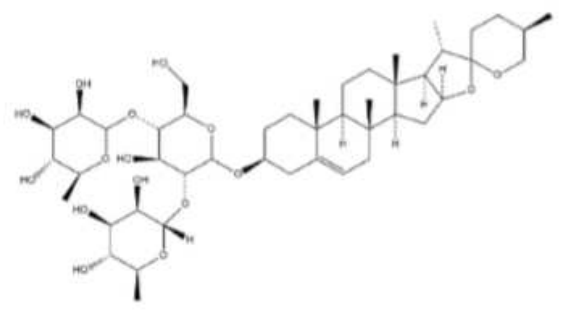

prosapogenin A

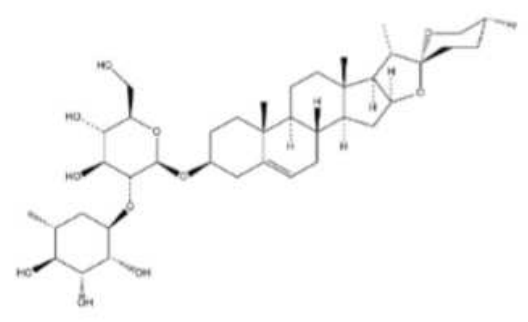

polyphillin $\mathrm{A}$

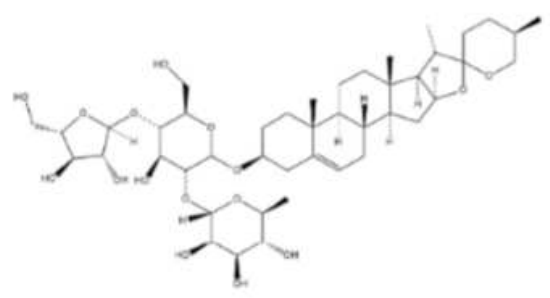

timosaponin A3

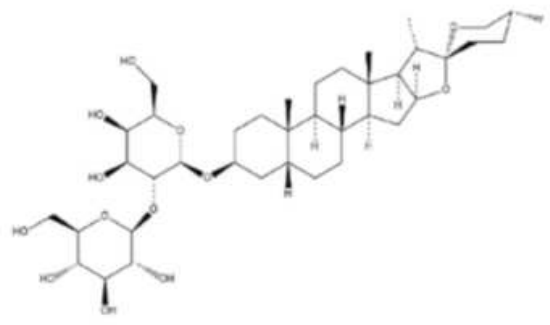

protodioscin

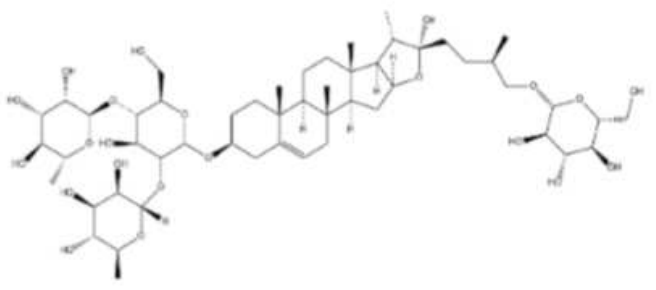

trillin

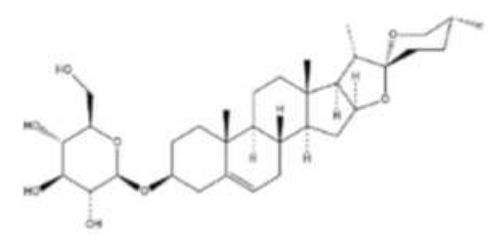

Figure 4

Diosgenin glycoside analogs. 


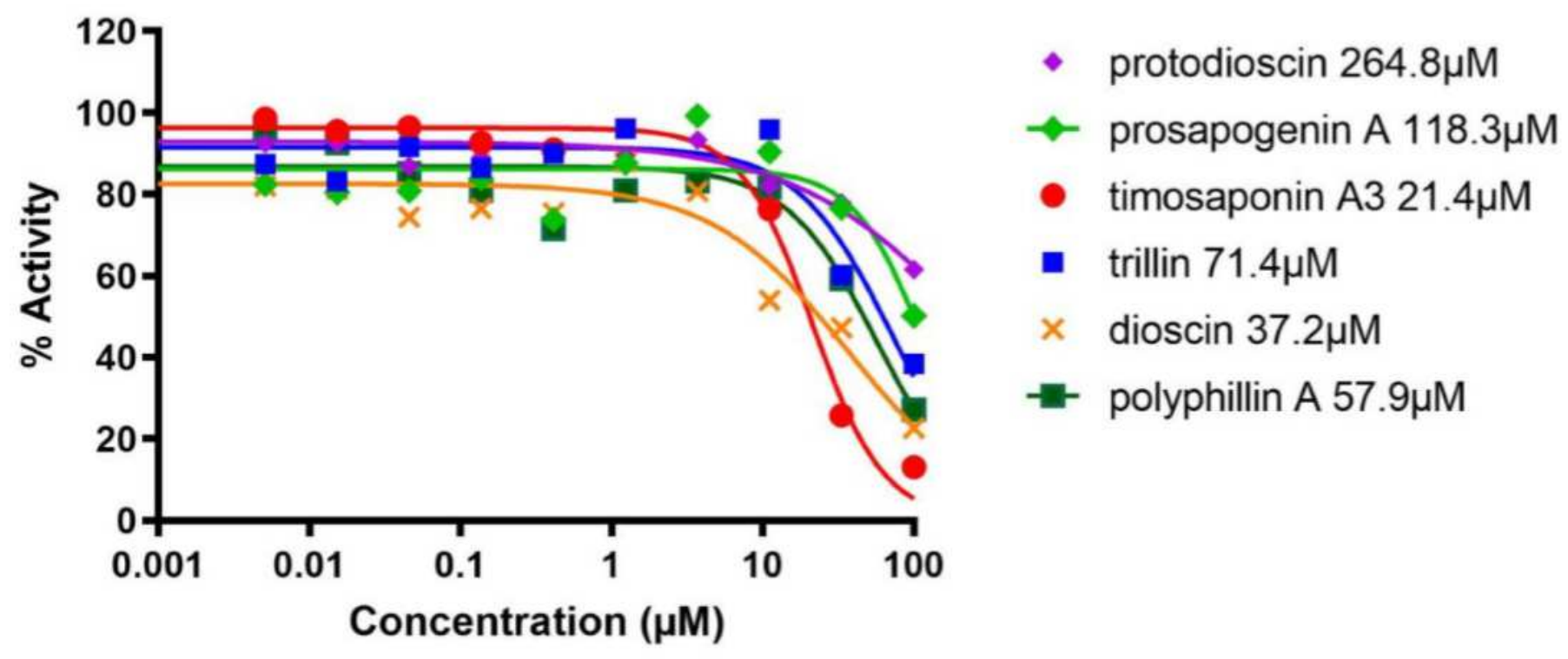

Figure 5

PIM3 kinase inhibition by diosgenin glycosides. PIM3 activity at a range of concentration of the indicated, with fitted curves and calculated IC50 values. 


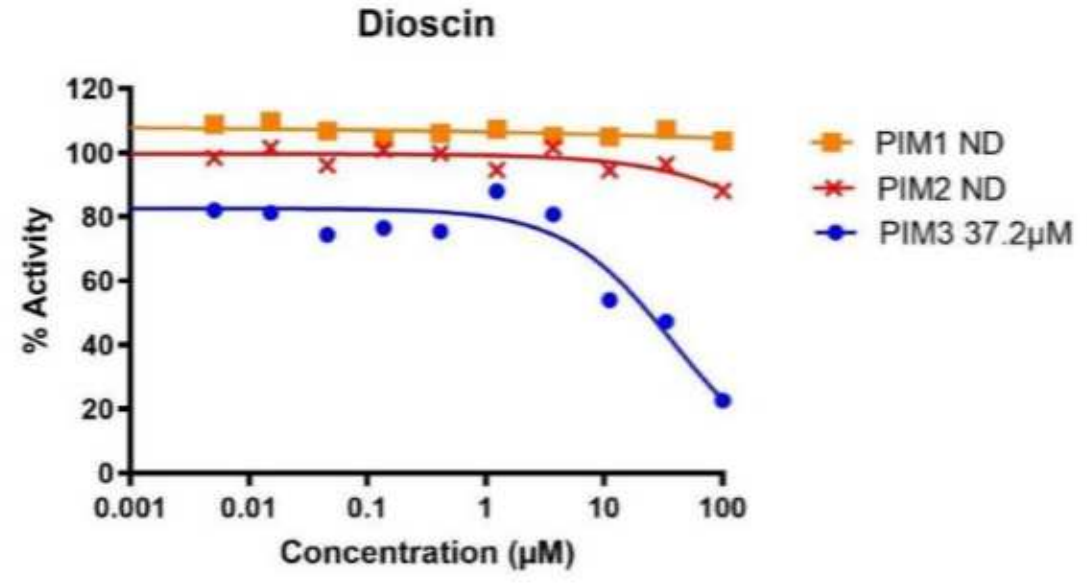

Timosaponin A3

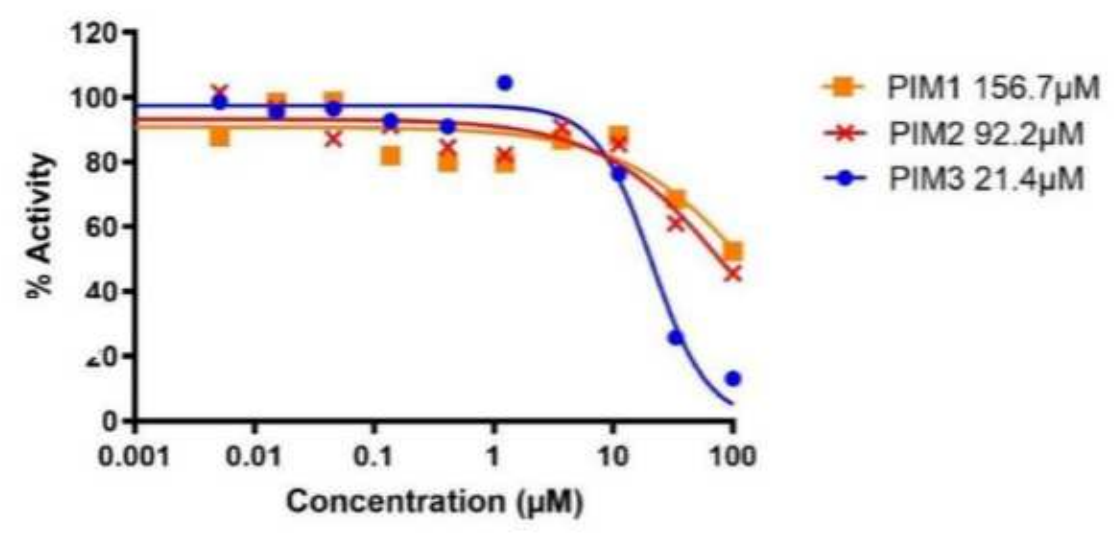

Polyphillin A

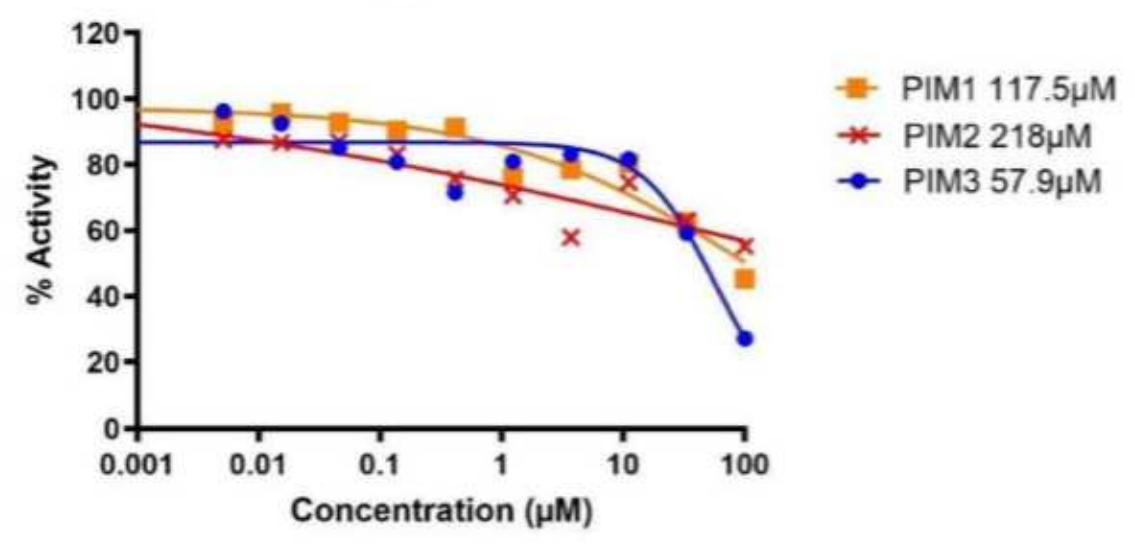

\section{Figure 6}

PIM kinase selectivity of diosgenin glycoside analogs. PIM1, 2, and 3 activity in the presence of a range of compound concentrations, with fitted curves and calculated IC50 values. ND= not determinable from the curve. 

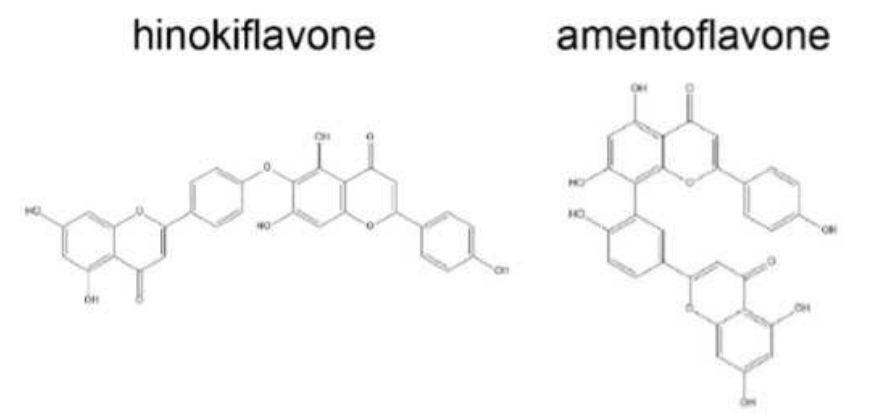

podocarpus flavone $A$ isoginkgetin

taiwanhomoflavone A 4'-O-methylochnaflavone
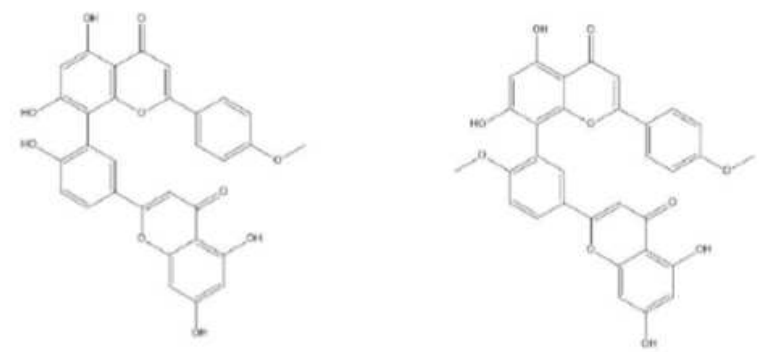

5',8" biluteolin tetrahydroamentoflavone
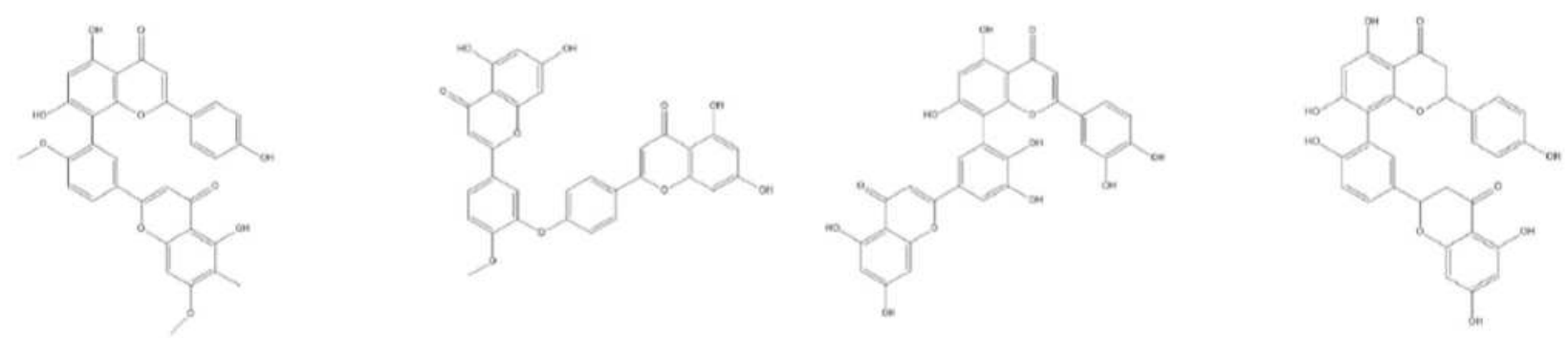

Figure 7

Biflavonoid analogs.

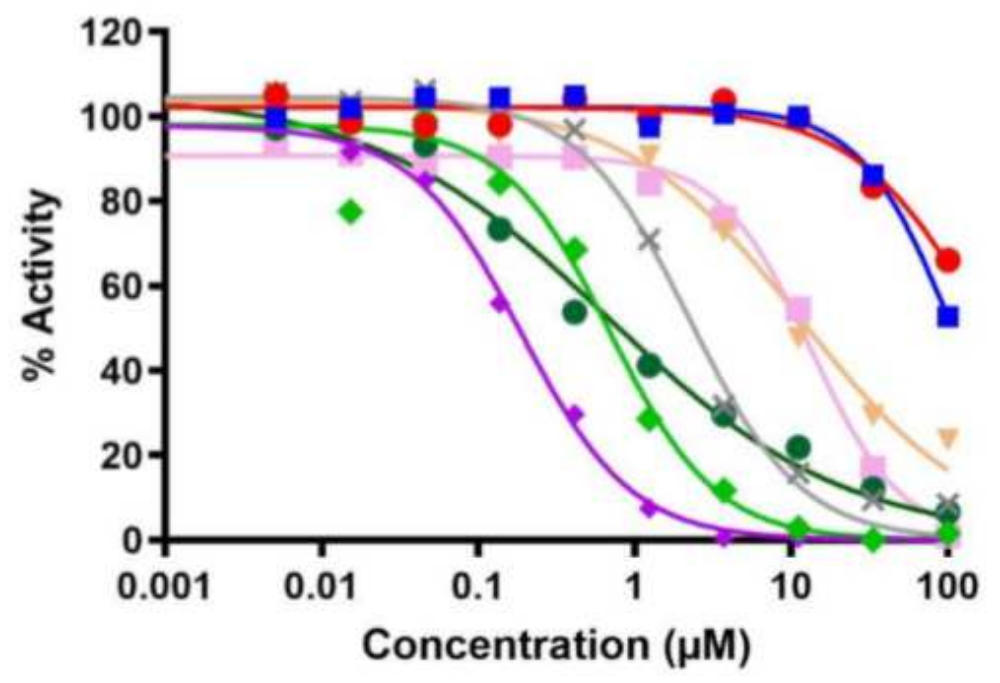

- amentoflavone $.188 \mu \mathrm{M}$

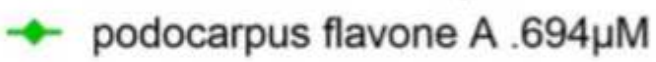

- isoginkgetin $171 \mu \mathrm{M}$

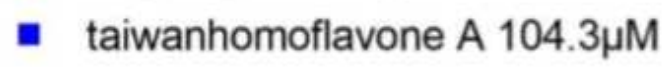

$\times$ hinokiflavone $2.23 \mu \mathrm{M}$

- $5^{\prime}, 8^{\prime \prime}$ biluteolin $.665 \mu \mathrm{M}$

- 4'-O-methylochnaflavone $11.63 \mu \mathrm{M}$

-n- tetrahydroamentoflavone $13.22 \mu \mathrm{M}$

Figure 8

PIM3 inhibition by biflavonoids. PIM3 activity at a range of concentration of the indicated, with fitted curves and calculated IC50 values. 

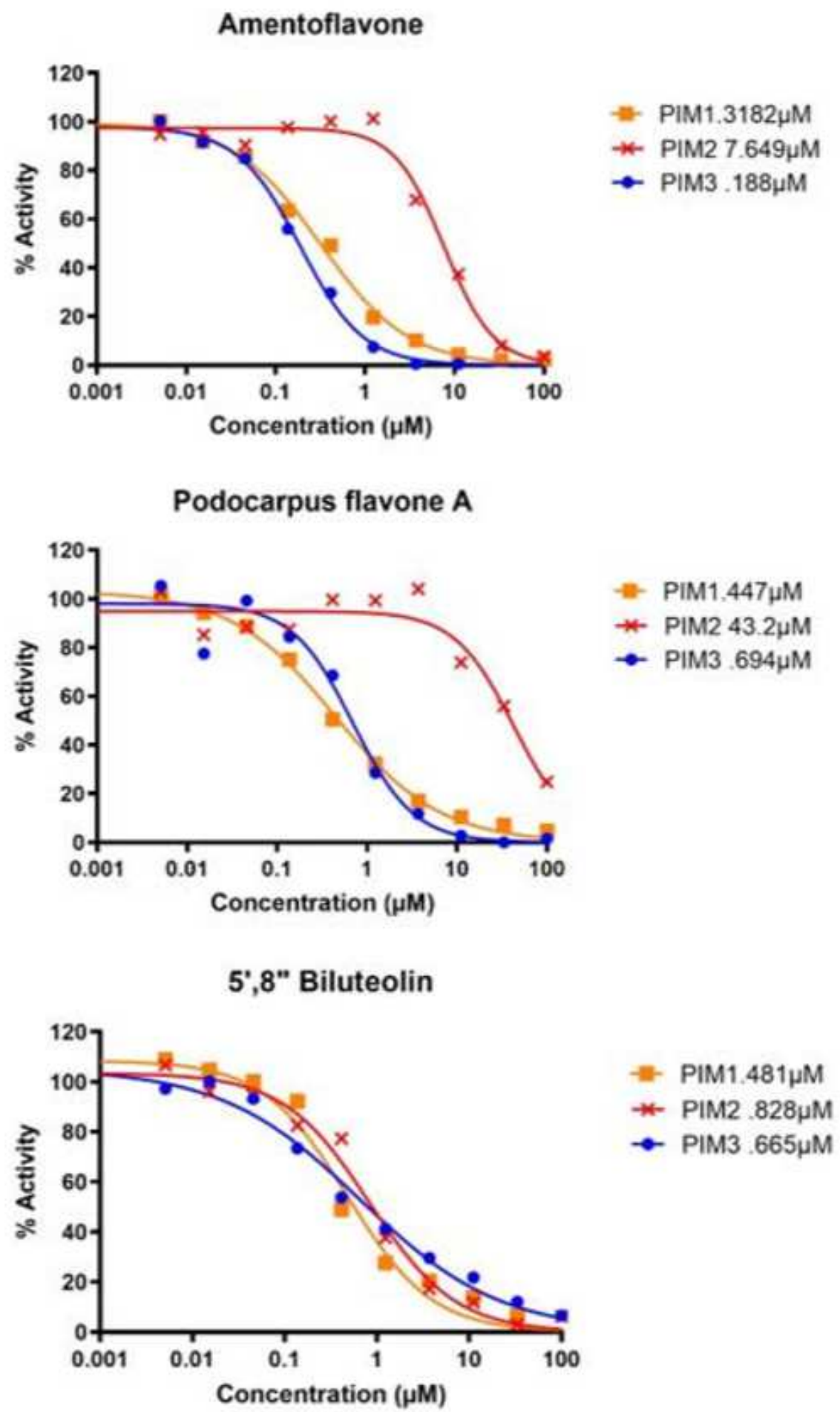

Figure 9

PIM kinase selectivity of biflavonoids. PIM1,2, and 3 activity in the presence of a range of compound concentrations, with fitted curves and calculated IC50 values. 